\title{
Retrato de familiares acompanhantes de crianças e adolescentes atendidos por retinoblastoma no Brasil: Estudo transversal
}

\author{
Portrayal of accompanying family members of children and teenagers cared for retinoblastoma in \\ Brazil: A cross-sectional study \\ Representación de familiares acompañantes de niños y adolescentes atendidos por retinoblastoma \\ en Brasil: Un estudio transversal
}

Recebido: 15/04/2021 | Revisado: 25/04/2021 | Aceito: 26/04/2021 | Publicado: 10/05/2021

Maria Izabel Pereira Messias
ORCID: https://orcid.org/0000-0002-0741-9221
Universidade Federal de Alfenas, Brasil
E-mail: maria.messias@ sou.unifal-mg.edu.br
Gabriela Silva Bochi
ORCID: https://orcid.org/0000-0003-1753-2655
Universidade Federal de Alfenas, Brasil
E-mail: gabriela.bochi@ @ou.unifal-mg.edu.br
Alicia Natali Ferreira da Silva
ORCID: https://orcid.org/0000-0002-3684-7831
Universidade Federal de Alfenas, Brasil
E-mail: alicia.silva@ sou.unifal-mg.edu.br
Fabiana Lucio de Oliveira
ORCID: https://orcid.org/0000-0003-2948-7556
E-mail: fabiana.lucio@ ifsuldeminas.edu.br
Jessica Luanda Lemos Melo
ORCID: https://orcid.org/0000-0002-9108-4312
Universidade Federal de Alfenas, Brasil
E-mail: jessica.melo@ sou.unifal-mg.edu.br
Namie Okino Sawada
ORCID: https://orcid.org/0000-0002-1874-3481
Universidade Federal de Alfenas, Brasil
E-mail: namie.sawada@ @unifal-mg.edu.br
Murilo César do Nascimento
ORCID: https://orcid.org/0000-0002-3436-2654
Universidade Federal de Alfenas, Brasil
E-mail: murilo.nascimento@ unifal-mg.edu.br

\begin{abstract}
Resumo
O retinoblastoma é o tumor intraocular mais frequente na infância. Não está claro quem são os familiares acompanhantes dos pacientes, onde obtêm informações sobre a doença, nem a sua satisfação com as orientações fornecidas pelos profissionais de saúde. Assim, este estudo objetivou retratar os familiares de crianças e adolescentes com diagnóstico ou histórico de retinoblastoma quanto à aspectos sociodemográficos, às fontes de informação utilizadas sobre a doença, à satisfação com as orientações recebidas por profissionais sobre tal câncer, bem como analisar se existe associação entre estas variáveis. Tratou-se de um estudo transversal, descritivo-analítico, via web survey no Brasil, a partir de mídias sociais e análise por estatística descritiva-inferencial. Participaram 129 pessoas, com média de idade de 34,61 anos, predominantemente mães, do lar, com onze anos de escolaridade, renda familiar de até um salário mínimo e residentes principalmente no sudeste brasileiro. Dos respondentes, 65,12\% afirmaram utilizar os sites da internet como fonte principal de informação. Quanto à satisfação com as orientações dos profissionais, predominaram notas elevadas, indicando alto contentamento com a atividade instrucional dos médicos e enfermeiros. Conclui-se, para além do retrato sociodemográfico alcançado, que os sites de internet foram a principal fonte de informação sobre o retinoblastoma e que houve alta satisfação referente às orientações fornecidas pelos profissionais. Houve associação entre a ocupação/profissão do lar do familiar com o uso de mídias sociais como fonte preferencial de informação sobre o retinoblastoma e também associação entre ser profissional autônomo e a satisfação com as orientações sobre a doença fornecidas pelos médicos.
\end{abstract}

Palavras-chave: Retinoblastoma; Família; Informação; Orientação; Neoplasias. 


\begin{abstract}
Retinoblastoma is the most common intraocular tumor in childhood. It is not yet clear who the accompanying family members of affected children and teenagers are, how they find out about the disease, or how satisfied they are with the guidance provided by healthcare providers. Thus, this study aimed to portray the family members of children and teenagers with retinoblastoma regarding their sociodemographic aspects, the sources of information used by them to learn about the disease, their satisfaction with the guidance received from healthcare providers regarding this form of cancer, and to analyze whether there is an association between these variables. It was a cross-sectional, descriptiveanalytical study, via web survey in Brazil, based on social media and analysis by descriptive-inferential statistics. A total of 129 people participated in this study, with an average age of 34.61 years, most of them were mothers, housewives, with eleven years of education, with a family income not exceeding minimum wage, and living mostly in the southeastern region of Brazil. Among the respondents, $65.12 \%$ of them stated that they use websites as their main source of information. Regarding the satisfaction with the guidance received from healthcare providers, there were scores above eight, indicating a high level of satisfaction with the instructional activity of physicians and nurses. It is concluded, in addition to the sociodemographic portrayal achieved, that websites emerged as the main source of information on retinoblastoma and that there was high satisfaction regarding the guidance received from healthcare providers. There was an association between family members whose occupation was considered to be that of a housewife and the use of social media as the main source of information regarding retinoblastoma, as well as an association between being self-employed and the satisfaction with the guidance provided by physicians regarding the disease.
\end{abstract}

Keywords: Retinoblastoma; Family; Information; Orientation; Neoplasms.

\title{
Resumen
}

El retinoblastoma es el tumor intraocular más común en la niñez. No está claro quiénes son los cuidadores familiares de los niños y adolescentes afectados, de dónde obtienen información sobre la enfermedad, ni su satisfacción con la orientación brindada por los profesionales de la salud. Así, este estudio tuvo como objetivo retratar a los familiares de niños y adolescentes con retinoblastoma en cuanto a aspectos sociodemográficos, las fuentes de información utilizadas sobre la enfermedad, la satisfacción con la orientación recibida por los profesionales sobre dicho cáncer, así como analizar si existe una asociación entre estas variables. Fue un estudio transversal, descriptivo-analítico, vía encuesta web en Brasil, basado en redes sociales y análisis por estadística descriptiva-inferencial. Participaron 129 personas, con una edad promedio de 34,61 años, predominantemente madres, del hogar, con once años de escolaridad, renta familiar de hasta un salario mínimo y residentes principalmente en la región sureste de Brasil. De los encuestados, el 65,12\% afirmó que utiliza sitios de Internet como principal fuente de información. Hubo altas calificaciones de satisfacción con la orientación de los profesionales, lo que indica un alto grado de satisfacción con las instrucciones de médicos y enfermeras. Se concluye, además del retrato sociodemográfico logrado, que: los sitios de internet fueron la principal fuente de información sobre el retinoblastoma; los familiares se mostraron satisfechos con la orientación brindada por los profesionales de la salud; Existió una asociación entre la ocupación / profesión del hogar del familiar con el uso de las redes sociales como fuente de información preferida sobre el retinoblastoma y también una asociación entre ser un profesional autónomo y la satisfacción con las pautas sobre la enfermedad proporcionadas por los médicos.

Palabras clave: Retinoblastoma; Familia; Información; Orientación; Neoplasias.

\section{Introdução}

O retinoblastoma é conhecido como o mais frequente dos tumores intraoculares na infância, correspondendo a $15 \%$ de todos os tumores que ocorrem no primeiro ano de vida (Assis Brasil et al., 2018; Palazzi et al., 2015). Neste contexto, a informação e a educação em saúde são extremamente importantes para a criança com câncer e para seus cuidadores/responsáveis/familiares que, devido à faixa etária na qual a doença mais incide, marcam fiel presença nos ambulatórios e hospitais. Quando ofertadas de forma clara, suficiente e em tempo oportuno, as informações/orientações sobre retinoblastoma possuem potencial para promover o diagnóstico precoce da doença, minimizar condições de ansiedade, estresse, medo do prognóstico, bem como sentimento de impotência e incerteza quanto ao futuro. No entanto, existem lacunas na comunicação entre profissionais de saúde e familiares e/ou responsáveis (Amador et al., 2018).

A justificativa desta pesquisa se refere à importância de adensar o conhecimento científico sobre a temática, bem como de se gerar informações para uma análise situacional com potencial de melhorar as atividades de informação e educação em saúde para familiares/responsáveis de pacientes com retinoblastoma. Acredita-se que para prover abordagens eficazes e estratégias esclarecedoras sobre a história natural da doença, as possibilidades terapêuticas, bem como o plano singular de 
cuidados, há primeiro que conhecer o perfil das pessoas, as fontes de informação acessadas por elas e a satisfação das orientações que recebem atualmente dos profissionais de saúde. Portanto, este estudo teve como objetivo retratar os familiares de crianças e adolescentes com diagnóstico ou histórico de retinoblastoma quanto à aspectos sociodemográficos, às fontes de informação utilizadas sobre a doença, à satisfação com as orientações recebidas por profissionais sobre tal câncer, bem como analisar se existe associação entre estas variáveis.

\section{Metodologia}

Estudo transversal, descritivo-analítico, via web survey, a partir de mídias sociais, que abordou familiares/acompanhantes de crianças e adolescentes atendidos por retinoblastoma no Brasil. Os critérios de inclusão foram: familiares/acompanhantes de ambos os sexos, idade igual ou superior a 18 anos. A seleção dos participantes foi por amostragem não-probabilística, do tipo acessibilidade ou por conveniência, no primeiro momento. Em seguida, os participantes tiveram a oportunidade de contribuir com o incremento da amostra por meio da técnica de bola de neve (Polit \& Beck, 2011).

O roteiro semiestruturado utilizado para a coleta de dados foi construído e refinado com o auxílio de três experts em: córnea, segmento anterior do olho e exames de imagem em oftalmologia; câncer de cabeça e pescoço, reabilitação do paciente cirúrgico e oncológico; e epidemiologia do câncer. Já para o estudo piloto contribuíram os 15 respondentes iniciais, os quais permaneceram na pesquisa como participantes.

Para a elaboração do roteiro semiestruturado de coleta foi utilizado o recurso Google Forms (Google, 2019). As seções do roteiro contemplaram o Termo de Consentimento Livre e Esclarecido - TCLE, critério de elegibilidade, características sociodemográficas dos acompanhantes, bem como perguntas referentes à satisfação com as orientações de profissionais da saúde e às fontes de informações sobre o retinoblastoma, na perspectiva dos acompanhantes.

A coleta de dados ocorreu entre março e maio de 2020, incluindo os 20 dias iniciais do estudo piloto. Tal etapa foi realizada mediante envio de link do roteiro semiestruturado via Google Forms, com resposta/autopreenchimento pelos participantes do estudo. $\mathrm{O}$ ambiente para acesso e convite aos participantes constituiu-se por grupos de apoio, associações e comunidade virtuais, existentes em redes sociais, bem como por grupos de contatos do aplicativo WhatsApp.

As respostas da web survey foram registradas de forma automática em planilha eletrônica do recurso Google Forms. Assim, os processos de dupla verificação e de tabulação dos dados deu espaço ao trabalho de normalização das variáveis numa planilha eletrônica. Ao se reconhecer a possibilidade de viés de seleção devido à amostragem por conveniência e bola de neve, bem como eventual viés de informação, por conta da estratégia web survey, a medida de controle possível aos pesquisadores foi garantir a devida atenção quanto à validade externa dos resultados.

Foi realizada análise descritiva dos dados, sendo os resultados apresentados e discutidos a partir da distribuição de frequências e das medidas de posição e de dispersão observadas. Já para analisar se havia associação entre os aspectos sociodemográficos e a satisfação com as orientações de profissionais da saúde, e as fontes de informações sobre o retinoblastoma acessadas pelos acompanhantes, procedeu-se dicotomizando as variáveis independentes/dependentes qualitativas politômicas nominais e ordinais segundo os agrupamentos de interesse.

Assim, a variável independente "escolaridade (do acompanhante)" foi recodificada em "possui quatro anos completos de estudo" (sim ou não); a variável independente "ocupação/profissão (do acompanhante)" foi recodificada para familiar sem trabalho" (sim ou não), "familiar autônomo" (sim ou não), "familiar com ocupação do lar" (sim ou não); e a variável independente "renda familiar (do acompanhante)" foi recodificada em "renda até um salário mínimo" (sim ou não).

Já a variável dependente "fonte principal de informação sobre o retinoblastoma" foi recodificada para "utiliza preponderantemente sites da internet - Google, etc. - e redes sociais/aplicativos - Facebook, Instagram, WhatsApp" (sim ou 
não), e "obtém informações principalmente de profissionais de saúde" (sim ou não). A outra variável dependente "satisfação com as orientações dos médicos em relação ao retinoblastoma" foi recodificada como "satisfação com a orientação médica igual ou maior que sete" (sim ou não). E a terceira variável dependente "satisfação com as orientações dos enfermeiros em relação ao retinoblastoma" foi recodificada em "satisfação com as orientações dos enfermeiros igual ou superior a sete" (sim ou não).

Devido à ausência de referência específica na literatura sobre os pontos de corte para as variáveis dependentes de interesse, esclarece-se que a escolha dos novos agrupamentos se deu de forma arbitrária, conforme conhecimento empírico dos pesquisadores. Contudo, o processo de recodificação em novas categorias dicotômicas foi pautado em princípios de bioestatística (Pagano \& Gauvreau, 2004).

O teste de normalidade para as variáveis numéricas foi feito com o teste de Shapiro-Wilk, sendo necessária a adoção de testes não paramétricos. As associações foram analisadas por meio do teste de qui-quadrado de Pearson e do teste exato de Fisher, bem como pela estimação da Razão de Prevalências (RP). Todos os testes foram realizados adotando-se 5\% de significância. Utilizou-se, para tal, o Software Statistical Package for the Sciences - SPSS. A discussão dos resultados foi feita por meio do diálogo entre os resultados obtidos, a literatura e o empírico/inferencial/autoral.

O trabalho foi aprovado pelo Comitê de Ética em Pesquisa da instituição proponente, conforme CAAE ${ }^{\circ}$ 24821819.3.0000.5142 e Parecer nº 3.698.834 de 12 de novembro de 2019.

\section{Resultados}

Participaram do estudo 129 familiares/acompanhantes de crianças e adolescentes com diagnóstico ou histórico de retinoblastoma. Em relação à distribuição dos participantes pelas regiões do Brasil, obteve-se mais repostas de pessoas da região Sudeste $(n=51,39,53 \%)$, seguida pelas regiões Sul $(n=29 ; 22,48 \%)$, Nordeste $(n=28 ; 21,70 \%)$, Centro-Oeste $(n=15$; $11,63 \%)$ e Norte $(\mathrm{n}=6 ; 4,65 \%)$.

A distribuição dos estados nos quais houve residentes respondentes é apresentada na Figura 1. 
Figura 1. Mapa com a distribuição dos estados de residência dos participantes do estudo, Brasil, 2020.

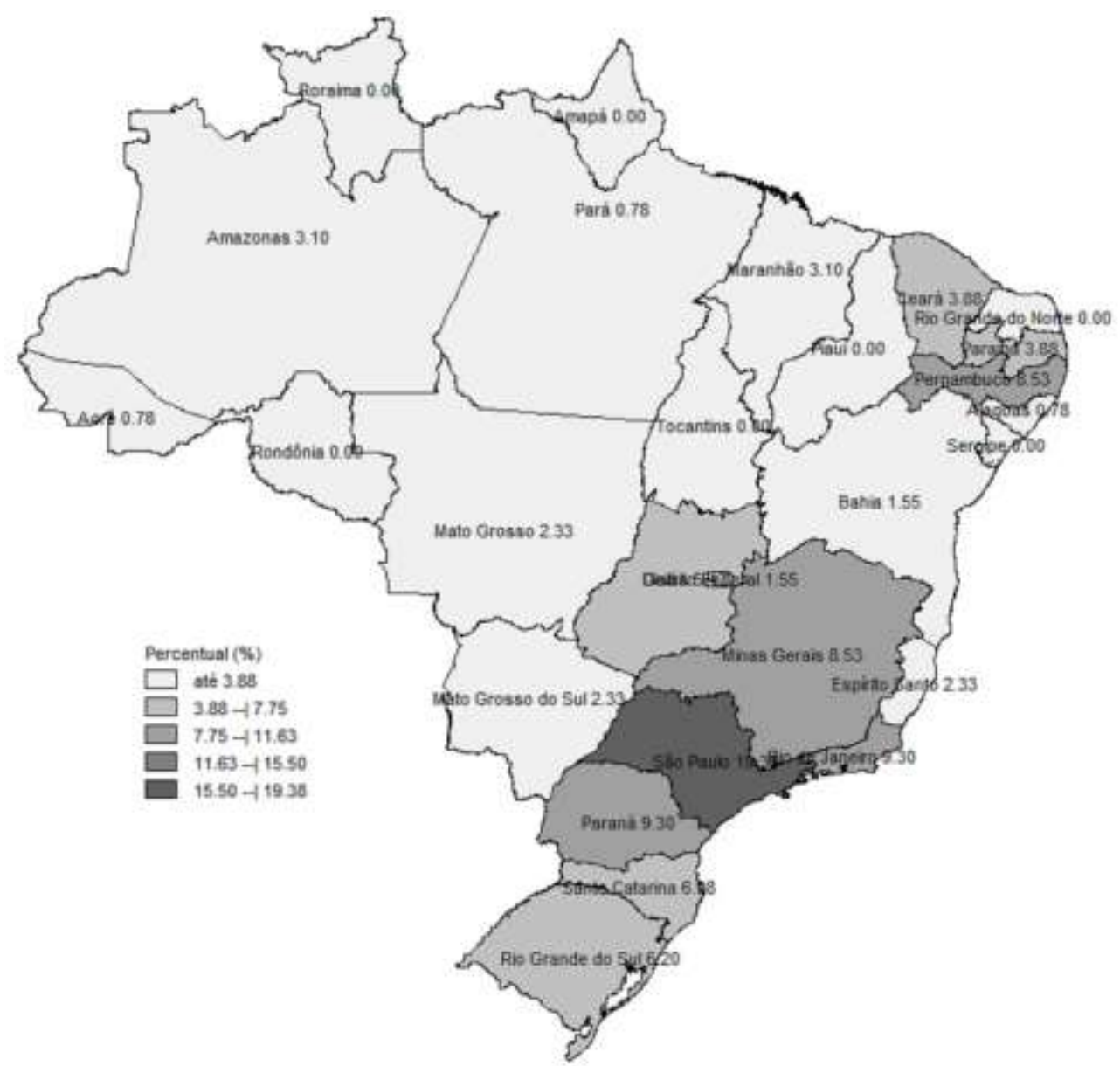

Fonte: Autores (2021)

Quanto aos aspectos sociodemográficos desses familiares, observou-se que a média de idade entre os acompanhantes foi de 34,61 anos (IC95\%: 22,00 - 53,00, mediana 35,00 anos, variância 42,22 e desvio padrão de 6,50).

Na Tabela 1 são apresentadas as variáveis sociodemográficas tipo de parentesco, sexo, faixa etária, escolaridade, profissão/ocupação e renda familiar dos familiares acompanhantes.

Tabela 1. Aspectos sociodemográficos dos familiares acompanhantes (N: $129=100 \%)$

\begin{tabular}{|c|c|c|c|}
\hline \multirow{2}{*}{\multicolumn{2}{|c|}{ Variáveis sociodemográficas dos familiares }} & \multicolumn{2}{|c|}{ Frequências } \\
\hline & & \multirow{2}{*}{$\frac{\mathbf{N}}{121}$} & \multirow{2}{*}{$\frac{\%}{93,80}$} \\
\hline \multirow{3}{*}{ Tipo de parentesco } & Mãe & & \\
\hline & Pai & 7 & 5,43 \\
\hline & Tio & 1 & 0,78 \\
\hline \multirow{2}{*}{ Sexo (acompanhante) } & Feminino & 121 & 93,80 \\
\hline & Masculino & 8 & 6,20 \\
\hline \multirow{4}{*}{ Faixa etária (acompanhante) } & 20 a 29 anos & 33 & 25,58 \\
\hline & 30 a 39 anos & 66 & 51,16 \\
\hline & 40 a 49 anos & 28 & 21,71 \\
\hline & 50 a 59 anos & 2 & 1,55 \\
\hline
\end{tabular}


Research, Society and Development, v. 10, n. 5, e38110515096, 2021

(CC BY 4.0) | ISSN 2525-3409 | DOI: http://dx.doi.org/10.33448/rsd-v10i5.15096

Tabela 1. Aspectos sociodemográficos dos familiares acompanhantes (N: $129=100 \%)$

(conclusão)

\begin{tabular}{|c|c|c|c|}
\hline \multirow{11}{*}{ Escolaridade (acompanhante) } & 0 anos / Não se aplica & 1 & 0,78 \\
\hline & Menos de 1 ano & 2 & 1,55 \\
\hline & 1 ano completo & 3 & 2,33 \\
\hline & 2 anos completos & 3 & 2,33 \\
\hline & 5 anos completos & 2 & 1,55 \\
\hline & 6 anos completos & 1 & 0,78 \\
\hline & 7 anos completos & 3 & 2,33 \\
\hline & 8 anos completos & 4 & 3,10 \\
\hline & 9 anos completos & 3 & 2,33 \\
\hline & 10 anos completos & 24 & 18,60 \\
\hline & 11 anos completos & 83 & 64,34 \\
\hline \multirow{13}{*}{ Profissão / Ocupação (acompanhante) } & Dona de casa / do lar & 29 & 22,48 \\
\hline & Autônoma & 12 & 9,30 \\
\hline & Analista & 5 & 3,88 \\
\hline & Assistente /Auxiliar Administrativa & 5 & 3,88 \\
\hline & Advogada & 5 & 3,88 \\
\hline & Professora & 5 & 3,88 \\
\hline & Desempregada & 4 & 3,10 \\
\hline & Enfermeira & 4 & 3,10 \\
\hline & Vendedora & 4 & 3,10 \\
\hline & Confeiteira & 4 & 3,10 \\
\hline & Administradora & 3 & 2,33 \\
\hline & Agricultora & 3 & 2,33 \\
\hline & Outras & 46 & 35,66 \\
\hline \multirow{10}{*}{ Renda familiar (acompanhante) } & Até 1 salário mínimo & 33 & 25,58 \\
\hline & Acima de 1 até 1,5 salário mínimo & 16 & 12,40 \\
\hline & Acima de 1,5 até 3 salários mínimos & 18 & 13,95 \\
\hline & Acima de 3 até 4,5 salários mínimos & 13 & 10,08 \\
\hline & Acima de 4,5 até 6 salários mínimos & 11 & 8,53 \\
\hline & Acima de 6 até 10 salários mínimos & 5 & 3,88 \\
\hline & Acima de 10 até 30 salários mínimos & 4 & 3,10 \\
\hline & Acima de 30 salários mínimos & 1 & 0,78 \\
\hline & Prefiro não informar & 21 & 16,28 \\
\hline & Não sei & 7 & 5,43 \\
\hline
\end{tabular}

Fonte: Autores (2021).

Em relação às fontes de informações sobre o retinoblastoma acessadas pelos acompanhantes, houve predomínio do uso dos sites da internet (Google, etc.), seguido dos profissionais da saúde e das redes sociais. A frequência das fontes de informações acessadas está resumida na Figura 2. 
Figura 2. Fontes de informações sobre o retinoblastoma acessadas pelos familiares acompanhantes, Brasil, 2020.

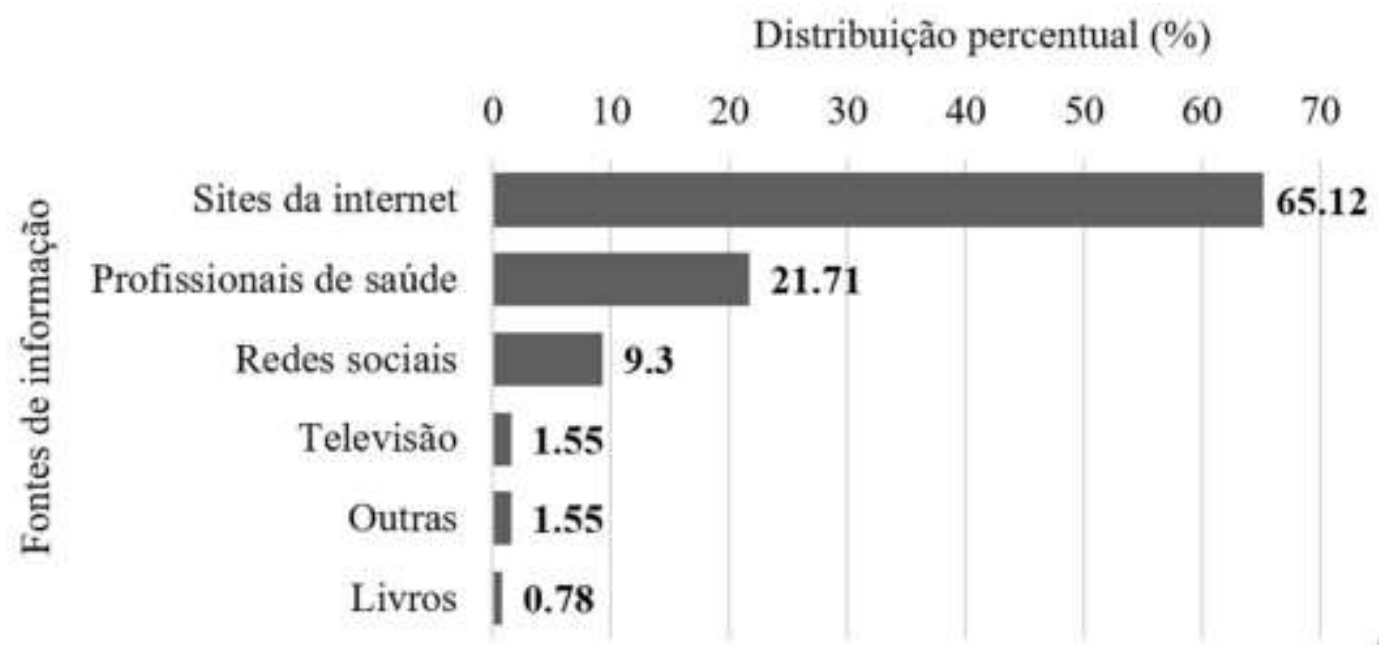

Fonte: Autores (2021).

No que diz respeito à satisfação dos acompanhantes com as orientações de profissionais da saúde sobre o retinoblastoma, os médicos receberam, em uma escala de 0 a 10, nota média de 9,24 (variância de 2,24 e desvio padrão de 1,49). Nesse sentido, 85 pessoas $(65,89 \%)$ avaliaram a sua satisfação com as orientações como 10,21 pessoas $(16,28 \%)$ deram 9 pontos e 11 participantes $(8,53 \%)$ atribuíram nota 8. Quanto aos enfermeiros as pontuações apresentaram média 8,87 (variância de 3,75 e desvio padrão de 1,93). Assim, 75 participantes avaliaram em $10(58,14 \%)$ a sua satisfação com as orientações recebidas sobre o retinoblastoma, 19 (14,73\%) deram nota 9 e 18 familiares (13,95\%) classificaram sua satisfação como nível 8. O detalhamento da distribuição das notas é apresentado na Figura 3.

Figura 3. Satisfação dos familiares de crianças e adolescentes com as orientações fornecidas sobre o retinoblastoma por médicos e enfermeiros, Brasil, 2020.

\section{—Satisfação com Médicos (\%) — —Satisfação com Enfermeiros (\%)}

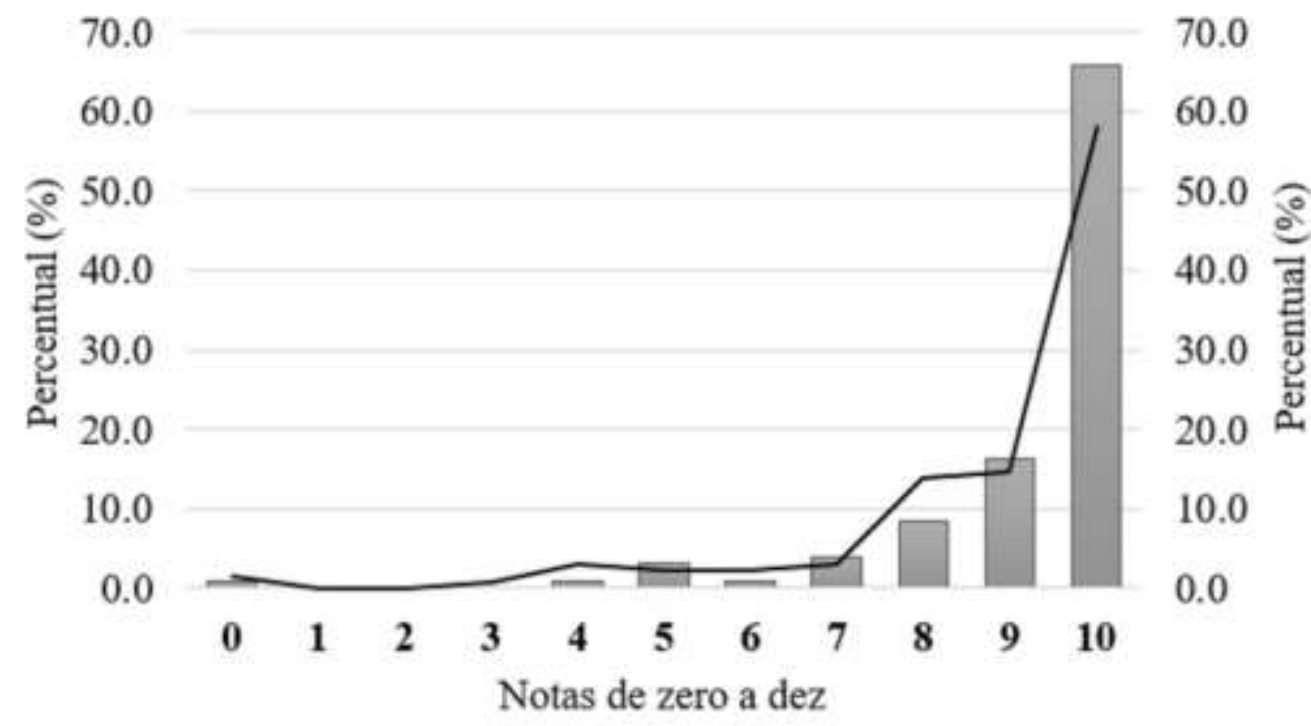

Fonte: Autores (2021).

No que se refere à verificação da existência de associação entre as características sociodemográficas e as fontes de informações sobre o retinoblastoma acessadas pelos familiares acompanhantes, observou-se que a ocupação/profissão do 
acompanhante do lar esteve associada à utilização de sites populares/redes sociais como fonte principal de informação sobre o retinoblastoma (teste de qui-quadrado de Pearson: $\mathrm{p}=0,033$ ). Quanto à força desta associação, a estimação da razão de prevalências (RP: 1,281; IC95\%: 1,072 - 1,531) sinalizou que o uso das homepages e redes sociais esteve 1,28 vezes mais presente dentre os familiares com ocupação do lar, quando comparados com ao uso destas mídias entre os familiares com as demais profissões.

Já quanto à verificação de associação entre os aspectos sociodemográficos e a satisfação dos familiares/acompanhantes com as orientações médicas sobre o retinoblastoma, a ocupação/profissão autônomo esteve associada à satisfação igual/maior a sete com as orientações dos médicos sobre o retinoblastoma (teste exato de Fisher: $\mathrm{p}=$ 0,027). Sobre a força de tal associação, a razão de prevalência (RP: 0,7835; IC95\%: 0,5639 - 1,089) sugeriu que a satisfação elevada com as orientações médicas sobre o referido câncer esteve 0,78 vezes menos presente dentre os familiares autônomos, quando comparado com a prevalência de alta satisfação dentre os acompanhantes que não eram autônomos. Contudo, atenção especial ao intervalo de confiança indicou magnitude de associação nula e, portanto, inexistência de diferença quanto à prevalência da satisfação elevada entre ambos os grupos.

\section{Discussão}

\subsection{Características sociodemográficas}

Foi possível conhecer características de familiares/acompanhantes de crianças e adolescentes atendidos por retinoblastoma oriundos das cinco grandes regiões do Brasil. O local de residência da criança ou adolescente com retinoblastoma impacta diretamente em questões como rapidez de diagnóstico e adesão ao tratamento. O tratamento para este tipo de câncer está concentrado em centros especializados, os quais podem estar distantes da população mais interiorana ou mesmo inacessíveis devido a questões socioeconômicas (Mattosinho et al., 2019).

Com relação à categorização dos participantes, o predomínio das mães como acompanhantes pode ser explicado pela questão cultural, em que o cuidado fica sob responsabilidade materna, além da perspectiva histórica de que a mãe deve se sacrificar pelos filhos e até outros familiares. Assim, o fato da maioria dos acompanhantes serem do sexo feminino converge para o elevado número de mães que acompanham seus filhos por ocasião de processos referentes à saúde-doença-assistência (Essuman et al., 2018; Mutti et al., 2018; Xiao et al., 2019).

No que se refere a idade média dos acompanhantes, um estudo envolvendo acompanhantes de crianças com retinoblastoma desenvolvido em Gana apresentou média de idade próxima da obtida no presente estudo, de 32,8 anos (Essuman et al., 2018). Levando em conta que a idade fértil das mulheres se alinha à essa faixa etária, é possível que as acompanhantes fossem mães jovens, já que o diagnóstico de retinoblastoma predomina em crianças com idade de até 5 anos.

Quanto à escolaridade dos acompanhantes, o presente estudo converge com a literatura, em que mais da metade dos acompanhantes possuem ensino médio completo. Esse é um dado importante, à medida que as informações que dizem respeito aos cuidados do paciente necessitam ser compartilhadas com os familiares acompanhantes, levando em consideração o nível de escolaridade. A baixa escolaridade prejudica a realização da capacitação dos acompanhantes com a intenção de treiná-los para assumirem a continuidade dos cuidados ao doente em casa. Quando identificado o baixo nível de escolaridade, o profissional de saúde deve procurar instruir o familiar sobre os cuidados com o doente, utilizando de métodos acessíveis, por meio de recursos que facilitem o processo ensino-aprendizagem (Kozan et al., 2016; Szareski \& Lana, 2009).

Sobre a profissão, a ocupação dona de casa/do lar se destacou em relação às demais. Em estudo semelhante, as profissões de maior frequência foram comerciantes e artesãos (Essuman et al., 2018). Naquela mesma pesquisa, a frequência de desempregados foi de 12,5\%, ao passo que, no presente trabalho, resultou em 3,10\%. As ocupações de dona de casa e desempregado permitem que o familiar se dedique a um acompanhamento mais próximo do paciente acometido por 
retinoblastoma. Durante o tratamento as crianças ou adolescentes necessitam de cuidado e acompanhamento diários, impossibilitando o acompanhante de seguir com suas atividades rotineiras, como o trabalho. A diária permanência dos acompanhantes no hospital resulta no seu envolvimento no cuidado, tornando-se parte da equipe, auxiliando na detecção de sinais de alterações do estado físico e emocional de seu familiar (Szareski \& Lana, 2009).

Foi observado que a expressiva parcela dos acompanhantes se alinhava às faixas predominantes de renda familiar mais baixas. Acredita-se que a baixa renda possa estar relacionada com o desemprego. Uma das situações que mais afeta a família vivendo o processo de doença de um de seus entes é a dificuldade financeira. Isso porque, além do significativo dano orçamentário, o adoecimento na família também pode implicar em prejuízo no trabalho, sobretudo quando é necessário se pensar em quem assumirá o papel de acompanhante do doente no hospital, requerendo seu afastamento laboral (IBGE, 2018; Szareski \& Lana, 2009).

\subsection{Fontes de informações, satisfação com as orientações de profissionais de saúde sobre o retinoblastoma e associações}

Com relação às fontes de informações sobre o retinoblastoma acessadas pelos acompanhantes, é possível observar uma predominância dos sites da internet. Outras pesquisas mostram que a criação desse meio de comunicação favoreceu bastante a democratização da informação e a troca de experiência entre pacientes (Azevedo Moretti et al., 2012). Os acompanhantes de pacientes com câncer que experimentam um déficit de suporte informacional de profissionais de saúde utilizam a internet como meio de buscar mais informação sobre sintomas, tratamentos e prognóstico (Dolce, 2011). Ademais, o suporte social aos acompanhantes de pacientes com câncer é necessário para ampliar a informação em saúde e o compartilhamento de experiências com outros acompanhantes e familiares, o que enriquece a compreensão da doença (Yuen et al., 2015).

Nota-se que a internet contribuiu para uma comunicação mais interativa e libertadora quanto às limitações individuais de espaço e tempo, tornando-a mais flexível (Vermelho et al., 2014). Assim, seu uso vem se constituindo em ferramenta importante no cotidiano das pessoas e, de modo especial, incrementando a divulgação de informações referentes à área da saúde (F. de Oliveira et al., 2013). Entretanto, o uso ampliado de sites como fonte de informação também preocupa, visto que parte das informações utilizadas pelos acompanhantes sobre doenças e o sobre seus tratamentos podem ter origem duvidosa. Neste sentido, a preocupação se assenta quanto à qualidade das informações, uma vez que certos portais podem difundir informações inadequadas, incompletas, incorretas e até prejudiciais (Azevedo Moretti et al., 2012; F. de Oliveira et al., 2013).

No contexto da educação em saúde, por exemplo, as redes sociais têm função relevante de informar o usuário. Neste sentido, os profissionais e cientistas se valem de tais mídias para fins educacionais, como o compartilhamento de informações sobre saúde-doença, por exemplo. Como as informações técnicas sobre saúde se tornaram mais populares e acessíveis por meio das mídias sociais, os artigos científicos disponibilizados podem disparar interação sobre os conteúdos em tempo real e sem o impedimento da distância (Greysen et al., 2015).

Assim, se por um lado o grande volume de informações disponibilizadas on-line é algo que preocupa, devido à possibilidade dos pacientes se depararem com conteúdo de qualidade questionável (Camargo \& Ito, 2012), por outro, a interação pelas mídias possibilita a troca de experiências entre usuários com problemas semelhantes e facilita o debate entre especialistas e pacientes. Há sinais de que o uso apropriado dos canais virtuais de comunicação/informação contribua até para melhorias na qualidade de vida dos usuários, maior autonomia, pró-atividade, e modificação benéfica da relação médicopaciente (Azevedo Moretti et al., 2012).

Já quanto à avaliação da satisfação com as orientações oferecidas pelos profissionais de saúde, questionar os indivíduos atendidos sobre tal contentamento é de suma importância pois auxilia no conhecimento sobre a qualidade de um tratamento e oferece subsídio aos trabalhadores e aos gestores da área da saúde (Brédart et al., 2002). Ademais, o sucesso do 
tratamento médico também é obtido por meio de uma boa adesão, o que está relacionado, dentre outros fatores, com a qualidade comunicativa do médico com o seu paciente (V. Z. de Oliveira \& Gomes, 2004).

Além disso, o principal vínculo entre o usuário e os serviços de saúde ocorre por intermédio dos enfermeiros, os quais conseguem promover a manutenção, a recuperação e a reabilitação da saúde do paciente, o que influencia a avaliação feita pelos usuários (Santos et al., 2017). A atuação da enfermagem facilita a aceitação do diagnóstico por parte da família e esse apoio é imprescindível visto que o diagnóstico de câncer frequentemente causa um choque, percebido pelo desespero dos pais que acreditam ser uma doença incurável, relacionando-a com a morte (Costa \& Ceolim, 2010). Ainda, o contato próximo entre pacientes e equipe multidisciplinar e o conhecimento disseminado sobre o retinoblastoma impactam positivamente nas taxas de sobrevivência das crianças e adolescentes, diante do tratamento do retinoblastoma (Traoré et al., 2018).

No presente estudo, parcela expressiva dos familiares/acompanhantes referiram alta satisfação com as orientações sobre o retinoblastoma obtidas pelos profissionais de saúde que os assistiram. Esse é um aspecto positivo aqui identificado e que diverge de outros achados da literatura. Um estudo realizado em um centro de referência de câncer do Sistema Único de Saúde brasileiro em Goiânia evidenciou um déficit de orientação por parte dos profissionais de saúde. Dos pacientes com tumores oculares malignos estudados, incluindo o retinoblastoma, poucos receberam apoio psicológico durante o tratamento ou informações sobre a reabilitação estética por meio de próteses oculares (Sirianni et al., 2013). Os pacientes que tiveram perda ocular e possuíam indicação para uso de prótese ocular relataram que desejariam que os profissionais de saúde orientassem e esclarecessem mais sobre o tratamento e apresentassem uma postura mais participativa (Nicodemo \& Ferreira, 2006).

Sabe-se que os profissionais de saúde possuem um papel fundamental em auxiliar os acompanhantes de pacientes com câncer na aquisição, no entendimento e na aplicação das informações de saúde (Yuen et al., 2015). Frequentemente os acompanhantes se sentem desinformados sobre o desenvolvimento da doença, exames e tratamentos. Sendo assim, deveria haver mais espaço para o esclarecimento das dúvidas e anseios da família (Amador et al., 2018).

Portanto, o conhecimento sobre o retinoblastoma deve ser amplamente difundido. Os familiares devem ser orientados a buscar ajuda médica precocemente, isso pode configurar num aspecto favorável para que se evite o atraso do diagnóstico e, em alguns casos, consequente perda de visão (Amador et al., 2018). Mesmo quando apenas os tratamentos conservadores não são mais suficientes e há indicação da remoção cirúrgica do globo ocular, a informação e a educação em saúde pelos profissionais de saúde e pelas mídias sociais ainda se fazem necessárias, uma vez que são estratégias potencializadoras de um cuidado qualificado e de um melhor prognóstico.

Identifica-se como limitações do estudo a amostra não probabilística adotada, bem como o fato de os participantes terem sido convidados a participar de forma exclusivamente virtual. Apesar de implicar em vantagens operacionais, logísticas e de tempo na coleta de dados, reconhece-se que a amostragem pela técnica de bola de neve e estratégia da web survey podem privilegiar uma seleção parcial e restrita do público alvo. Assim, a taxa de retorno heterogênea sob a perspectiva geográfica, atrelada à falta de garantias sobre o acesso da população de interesse às tecnologias on-line, contas em redes sociais e aplicativo de comunicação virtual empregado, são destacados como fatores não controlados que podem interferir na validade externa da pesquisa. Contudo, os resultados ora apresentados, apesar de não generalizáveis para todo o Brasil, configuram um importante retrato que representa parcialmente os familiares acompanhantes de crianças e adolescentes atendidos por retinoblastoma no país.

Ademais, embora o tipo de estudo ora apresentado não possibilite determinar nem discutir causalidade, uma vez que em estudos transversais o critério da temporalidade não pode ser aplicado, acredita-se que, atendendo a uma das vantagens metodológicas das abordagens seccionais, esse trabalho contribuiu na elaboração de uma hipótese que necessita de futura investigação e esclarecimento. À saber: a ocupação/profissão do lar do familiar é condicionante ou determinante para o uso de mídias sociais como fonte principal de informação sobre o retinoblastoma. Diante disso, sugere-se que novas pesquisas sejam 
realizadas em populações maiores e por meio de delineamentos longitudinais, com a finalidade de testar tal hipótese quanto ao possível efeito e nexo causal.

\section{Conclusão}

Os familiares/acompanhantes das crianças e adolescentes atendidos por retinoblastoma foram retratados, de forma predominante, como pessoas do sexo feminino, com média de idade igual a 34,61 anos, sendo mães, com alta escolaridade, baixa renda, ocupação dona de casa e local de residência mais frequente no estado brasileiro de São Paulo.

Quanto à principal fonte de informação sobre o retinoblastoma, observou-se frequência mais expressiva do uso de sites da internet pelos familiares. Com relação à satisfação dos acompanhantes sobre as orientações fornecidas por médicos e enfermeiros sobre o tal câncer, foram identificadas predomínio de notas acima de oito, sinalizando para alta satisfação referente às informações recebidas por tais profissionais.

O estudo também contribuiu com a hipótese de que a ocupação do lar do familiar é determinante/condicionante para o uso de mídias sociais como fonte principal de informação sobre o retinoblastoma. Contudo, tal relação necessita ser mais bem elucidada, motivo pelo qual sugere-se que sejam desenvolvidas outras investigações com outros delineamentos, cenários e populações sobre a temática do retinoblastoma no Brasil e no mundo.

\section{Agradecimentos}

Registra-se sincera gratidão a todos os familiares acompanhantes das crianças e adolescentes acometidos por retinoblastoma que, de forma voluntária, atenciosa e gentil, participaram da pesquisa e possibilitaram o presente artigo.

\section{Referências}

Amador, D. D., Marcílio, A. C., Soares, J. dos S. S., Marques, F. R. B., Duarte, A. M., \& Mandetta, M. A. (2018). The strength of information on retinoblastoma for the family of the child. Acta Paul. Enferm. (Online), 31(1), 87-94.

Assis Brasil, E. S. de, Bencke, E. L., Canevese, F. F., \& Romani, F. A. (2018). Retinoblastoma: diagnostic evaluation and treatment update. Acta méd. (Porto Alegre), 39(2), 402-415.

Azevedo Moretti, F., Elias de Oliveira, V., \& Koga da Silva, E. M. (2012). Access to health information on the internet: a public health issue? Revista da Associação Médica Brasileira, 58(6), 650-658.

Brédart, A., Razavi, D., Robertson, C., Brignone, S., Fonzo, D., Petit, J.-Y., \& de Haes, J. C. J. M. (2002). Timing of patient satisfaction assessment: effect on questionnaire acceptability, completeness of data, reliability and variability of scores. Patient Education and Counseling, 46(2), $131-136$.

Camargo, A. de, \& Ito, M. (2012). Utilização das Tecnologias de Informação e Comunicação na área da saúde: uso das redes sociais pelos médicos. Journal of Health Informatics, 4(4), 165-169.

Costa, T. F. da, \& Ceolim, M. F. (2010). Nursing in palliative care for children and adolescent with cancer. Revista Gaúcha de Enfermagem, 31(4), 776-784.

Dolce, M. C. (2011). The Internet as a source of health information: experiences of cancer survivors and caregivers with healthcare providers. Oncology Nursing Forum, 38(3), 353-359.

Essuman, V. A., Braimah, I. Z., Ndanu, T. A., \& Ntim-Amponsah, C. T. (2018). Presentation of children with advanced retinoblastoma for treatment in Ghana: the caretakers perspectives. West Afr J Med, 35(1), 9-14.

Google. (2019). Google Forms. https://www.google.com/intl/pt-BR/forms/about/

Greysen, S. R., Arora, V. M., \& Auerbach, A. D. (2015). Peer-reviewed Publications in the Era of Social Media - JHM 2.0. Bone, 9(4), 269-270.

IBGE. (2018). Synthesis of social index: An analysis of the living standars of the Brazilian population 2018. IBGE.

Kozan, L., Claudia, A., Wanderbroocke, N. S., \& Polli, G. M. (2016). Social Support Among Escorts of Children Hospitalized in a Hematopediatrics Unit. $14(1), 53-78$.

Mattosinho, C. C. D. S., Moura, A. T. M. S., Oigman, G., Ferman, S. E., \& Grigorovski, N. (2019). Time to diagnosis of retinoblastoma in Latin America: A systematic review. Pediatric Hematology and Oncology, 36(2), 55-72.

Mutti, C. F., Cruz, V. G. da, Santos, L. F., Araújo, D. de, Cogo, S. B., \& Neves, E. T. (2018). Clinical and Epidemiological Profile of Children and 
Research, Society and Development, v. 10, n. 5, e38110515096, 2021

(CC BY 4.0) | ISSN 2525-3409 | DOI: http://dx.doi.org/10.33448/rsd-v10i5.15096

Adolescentes with Cancer in an Oncology Service. Rev. Bras. Cancerol, 64(3), 293-299.

Nicodemo, D., \& Ferreira, L. M. (2006). Questionnaire of the psychosocial profile of the patient with anophthalmia with indication of ocular prosthesis. Arq Bras Oftalmol, 69(4), 463-470.

Oliveira, F. de, Goloni-Bertollo, E. M., \& Pavarino, É. C. (2013). A Internet como fonte de Informação em Saúde. Journal of Health Informatics, 5(3), 98102.

Oliveira, V. Z. de, \& Gomes, W. B. (2004). Doctor-patient communication and adhesion to treatment in adolescents with chronic organic diseases. Estudos de Psicologia (Natal), 9(3), 459-469.

Pagano, M., \& Gauvreau, K. (2004). Princípios de bioestatística. Cengage Learning.

Palazzi, M. A., Abreu, H. F. H. de, Freitas, A. C. L. H. de, Quagliato, L. B., \& Freitas, J. A. H. de. (2015). Optical coherence tomography in the evaluation of macular retinoblastoma. Rev. bras. oftalmol, 74(5), 275-278.

Polit, D. F., \& Beck, C. T. (2011). Fundamentos de Pesquisa em Enfermagem: Avaliação de Evidências para a Prática da Enfermagem (7ºd). Artmed.

Santos, M. A. dos, Sardinha, A. H. de L., \& Santos, L. N. dos. (2017). User satisfaction with the care of nurses. Revista Gaúcha de Enfermagem, 38(1), e57506.

Sirianni, D., Leles, C. R., \& Mendonça, E. F. (2013). A 12-year retrospective survey of management of patients with malignant neoplasms in the orbital cavity in a brazilian cancer hospital. The Open Dentistry Journal, 7, 140-145.

Szareski, C., \& Lana, L. D. (2009). Profile of Family Companions : Contributions To Nursing Educational Actions. 13(1), $28-33$.

Traoré, F., Sylla, F., Togo, B., Kamaté, B., Diabaté, K., Diakité, A. A., Diall, H., Dicko, F., Sylla, M., Bey, P., Desjardins, L., Gagnepain-Lacheteau, A., Coze, C., Harif, M., \& Doz, F. (2018). Treatment of retinoblastoma in Sub-Saharan Africa: Experience of the paediatric oncology unit at Gabriel Toure Teaching Hospital and the Institute of African Tropical Ophthalmology, Bamako, Mali. Pediatric Blood \& Cancer, 65(8), e27101.

Vermelho, S. C., Velho, A. P. M., Bonkovoski, A., \& Pirola, A. (2014). Refletindo sobre as redes sociais digitais. Educacao e Sociedade, 35(126), 179-196.

Xiao, W., Ye, H., Zeng, H., Tang, L., Chen, R., Gao, Y., Mao, Y., \& Yang, H. (2019). Associations among Socioeconomic Factors, Lag Time, and High-Risk Histopathologic Features in Eyes Primarily Enucleated for Retinoblastoma. Current Eye Research, 44(10), 1144-1149.

Yuen, E. Y. N., Innovation, P. H., \& Health, P. (2015). Development of a conceptual model of cancer caregiver health literacy. European Journal of Cancer Care, 25(2), 294-306. 